\title{
Osteomielite crônica mandibular em pacientes pediátricos
}

\author{
Chronic mandibular osteomyelitis in pediatric patients \\ Osteomielitis mandibular crónica en pacientes pediátricos \\ Matheus Francisco Barros RODRIGUES ${ }^{1}$ \\ Layla Louise de Amorim ROCHA ${ }^{2}$ \\ Cristofe Coelho Lopes da ROCHA ${ }^{3}$ \\ Sérgio Takashi KUSSABA ${ }^{4}$ \\ ${ }^{1,2}$ Curso de Odontologia, Faculdade Cathedral, 69307-053 Boa Vista - RR, Brasil \\ ${ }^{3}$ Departamento infraestrutura, Instituto Federal, 69303-340 Boa Vista - RR, Brasil \\ ${ }^{4}$ Departamento de Cirurgia e Traumatologia Bucomaxilofacial, Hospital da Criança Santo Antônio, 69308-160 Boa Vista - RR, Brasil
}

\section{Resumo}

A osteomielite é considerada uma doença incomum em pacientes saudáveis e de difícil diagnóstico e tratamento. Estudos sugerem que patologias infecciosas periodontais e peri-implantares como gengivites, periodontites e peri-implantites atuam como fatores mais comuns, predisponentes para osteomielites dos maxilares. Osteomielites crônicas exigem tratamento com cobertura antibiótica e procedimentos cirúrgicos. O tratamento envolve avaliação, determinação da etiologia, terapia antimicrobiana, desbridamento da lesão, remoção dos sequestros ósseos e decorticação óssea associada ao emprego sistêmico de antimicrobianos, geralmente de amplo espectro. O presente trabalho tem como objetivo relatar dois casos clínicos de osteomielite crônica infantil, bem como realizar uma análise comparativa de casos clínicos já publicados em artigos científicos. Pôde-se concluir que a associação de características clínicas, exames histopatológicos e achados radiográficos podem reunir características comuns para diversos tipos de osteomielite. Portanto, devem culminar em fatores contribuintes para o diagnóstico final. Em ambos os casos relatados, o tratamento foi eficaz utilizando terapia medicamentosa com o uso de antiinflamatórios e antibióticos aliados a tratamento cirúrgico que consistiu em desbridamento da lesão. Ambos os pacientes foram acompanhados e proservados, não havendo recidiva.

Descritores: Osteomielite; Antibacterianos; Patologia Bucal; Procedimentos Cirúrgicos Bucais.

\section{Abstract}

Osteomyelitis is considered an unusual disease in healthy patients and is difficult to diagnose and treat. Research suggests that infectious periodontal and implant-related diseases such as gingivitis, periodontitis and implant inflammations act as the most common factors in terms of a predisposition to jawbone osteomyelitis. Chronic osteomyelitis requires treatment with antibiotics and surgical procedures. The treatment process requires an assessment, etiology determination, antimicrobial therapy, injury debridement, removal of bone sequestrations and bone dicortication associated with the systemic use of antimicrobial agents, generally broad spectrum agents. This paper intends to report two clinical cases of chronic ostheomyelitis in children, as well as to perform a comparative analysis of clinical cases that have already been published in scientific articles. One may conclude that the association of clinical characteristics, histopathological tests and radiography findings can gather common characteristics for multiple types of osteomyelitis. Therefore, they should culminate with contributing factors for a final diagnosis. In both reported cases, treatment was effective in using drug therapy with anti-inflammatory and antibiotic agents paired with surgical treatment consisting of injury debridement. Both patients were monitored and prospectively observed, there being no relapse.

Descriptors: Osteomyelitis; Anti-Bacterial Agents; Pathology, Oral; Oral Surgical Procedures.

\section{Resumen}

La osteomielitis se considera una enfermedad poco común en pacientes sanos y es difícil de diagnosticar y tratar. Los estudios sugieren que las patologías infecciosas periodontales y periimplantarias como la gingivitis, periodontitis y periimplantitis actúan como los factores más comunes, lo que predispone a la osteomielitis de la mandíbula. La osteomielitis crónica requiere tratamiento con cobertura antibiótica y procedimientos quirúrgicos. El tratamiento implica la evaluación, la determinación de la etiología, la terapia antimicrobiana, el desbridamiento de la lesión, la eliminación del secuestro óseo y la decorticación ósea asociada con el uso sistémico de antimicrobianos, generalmente de amplio espectro. Este artículo tiene como objetivo informar dos casos clínicos de osteomielitis crónica infantil, así como realizar un análisis comparativo de casos clínicos ya publicados en artículos científicos. Se concluyó que la asociación de características clínicas, exámenes histopatológicos y hallazgos radiográficos pueden reunir características comunes para varios tipos de osteomielitis. Por lo tanto, deben culminar en factores contribuyentes al diagnóstico final. En ambos casos informados, el tratamiento fue efectivo con terapia farmacológica con antiinflamatorios y antibióticos combinados con tratamiento quirúrgico que consistió en el desbridamiento de la lesión. Ambos pacientes fueron seguidos y mantenidos, sin recurrencia.

Descriptores: Osteomielitis; Antibacterianos; Patología Bucal; Procedimientos Quirúrgicos Orales.

\section{INTRODUÇÃO}

A osteomielite corresponde a uma inflamação do osso medular ${ }^{1}$, sendo um processo inflamatório acompanhado de destruição óssea, causado por um microrganismo infectante, podendo ser limitada a uma porção do osso ou envolver várias regiões, como medula, periósteo e tecidos moles circundantes ${ }^{2}$. É considerada uma doença incomum em pacientes saudáveis e de difícil diagnóstico e tratamento ${ }^{3}$.

Geralmente a área mais acometida pela patologia é a mandíbula por não possuir rico suprimento sanguíneo, fornecido apenas por meio da artéria alveolar inferior ${ }^{1}$. A ocorrência nos maxilares é menor devido ao seu rico suprimento sanguíneo, derivado de diversas artérias ${ }^{1}$.
Estudos
sugerem
que patologias

infecciosas periodontais e peri-implantares como gengivites, periodontites e peri-implantites atuam como fatores mais comuns, predisponentes para osteomielites dos maxilares ${ }^{4}$. Os parâmetros de diagnóstico de osteomielite são baseados nos achados clínicos da história inicial, exame físico e exames laboratoriais ${ }^{5}$. Ao observar características clínicas de osteomielite são solicitados ao paciente exames de radiografia panorâmica, tomografia computadorizada, laboratoriais e histopatológico ${ }^{6,7}$.

Os exames de imagem característicos da patologia demonstram imagens radiopacas mal delimitadas de demarcação difusa e irregular com aspecto de ruído de traça ${ }^{8}$. Ao realizar análise histopatológica pode-se identificar em áreas de esclerose várias trabéculas irregulares de osso 
pagetóide, bordas osteoblásticas e áreas focais de atividade osteoclástica ${ }^{7}$. As amostras ósseas mostram lesões ósseas reativas, caracterizadas por remodelação do osso cortical e subcortical, além da formação de osso subperiosteal ${ }^{9}$.

Osteomielites crônicas exigem tratamento com cobertura antibiótica e procedimentos cirúrgicos agressivos $^{1}$. O tratamento envolve avaliação, determinação da etiologia, terapia antimicrobiana, desbridamento da lesão, remoção dos sequestros ósseos e descorticação óssea associada ao emprego sistêmico de antimicrobianos, geralmente de amplo espectro $^{5,10}$. As medicações antibióticas de escolha são a clindamicina, as penicilinas e as fluoroquinolonas, por serem efetivas contra infecções odontogênicas e terem um grande poder de penetração óssea ${ }^{1}$.

$\mathrm{Na}$ área acometida há comprometimento da circulação e, por conta disso, o paciente tem que ser internado para receber altas doses de antibiótico intravenoso a fim de controlar inicialmente os sintomas ${ }^{1}$. Além desses, reserva-se os corticosteróides, bisfosfonatos ou imunomoduladores para os casos de recidivas múltiplas ${ }^{11}$.

O presente trabalho tem como objetivo relatar dois casos clínicos de osteomielite crônica infantil, bem como realizar uma análise comparativa de casos clínicos já publicados em artigos científicos.

\section{CASO CLÍNICO}

\section{- Caso Clínico 1}

Paciente do gênero masculino, 8 anos de idade, procurou o Hospital da Criança Santo Antônio relatando queixas álgicas em região mandibular. Ao exame clínico foi observada a assimetria facial com expansão da cortical óssea vestibular, de consistência endurecida, localizada na altura dos dentes 85 e 46, além de processo inflamatório com sinais flogísitcos, levando à hipótese diagnóstica de osteomielite crônica e displasia fibrosa. Foi solicitada tomografia computadorizada (Figura 1), onde foi possível observar lesão na raiz dentária do primeiro molar mandibular à direita. Caracteriza-se por foco hipoatenuante envolvendo a raiz, podendo estar relacionado a abscesso dentário, na dependência de correlação clínica, formando trajetos determinando descontinuidade da cortical e com extensão à face anterior/externa junto ao osso mandibular e contida por uma importante reação periosteal laminar contínua e espessa, por um extensor de segmento estimado em aproximadamente $3,8 \mathrm{~cm}$ e espessura de até $1,0 \mathrm{~cm}$. Observa-se também reação laminar de aspecto filiforme na face interna da mandibular neste nível, com espessura de até $0,2 \mathrm{~cm}$. Associa-se lesão na coroa dentária neste nível. Foi realizada biópsia incisional com intenção diagnóstica. $\mathrm{O}$ exame anatomopatológico como pode ser visto na Figura 2, desmonstrou os múltiplos fragmentos provenientes de curetagem, apresentando tecido ósseo vital com várias trabéculas $\mathrm{e}$ tecido conjuntivo fibroso entremeando essas trabéculas. Nota-se também intenso infiltrado inflamatório crônico associado. Os aspetos clínicos e microscópicos favoreceram o diagnóstico de osteomielite crônica.

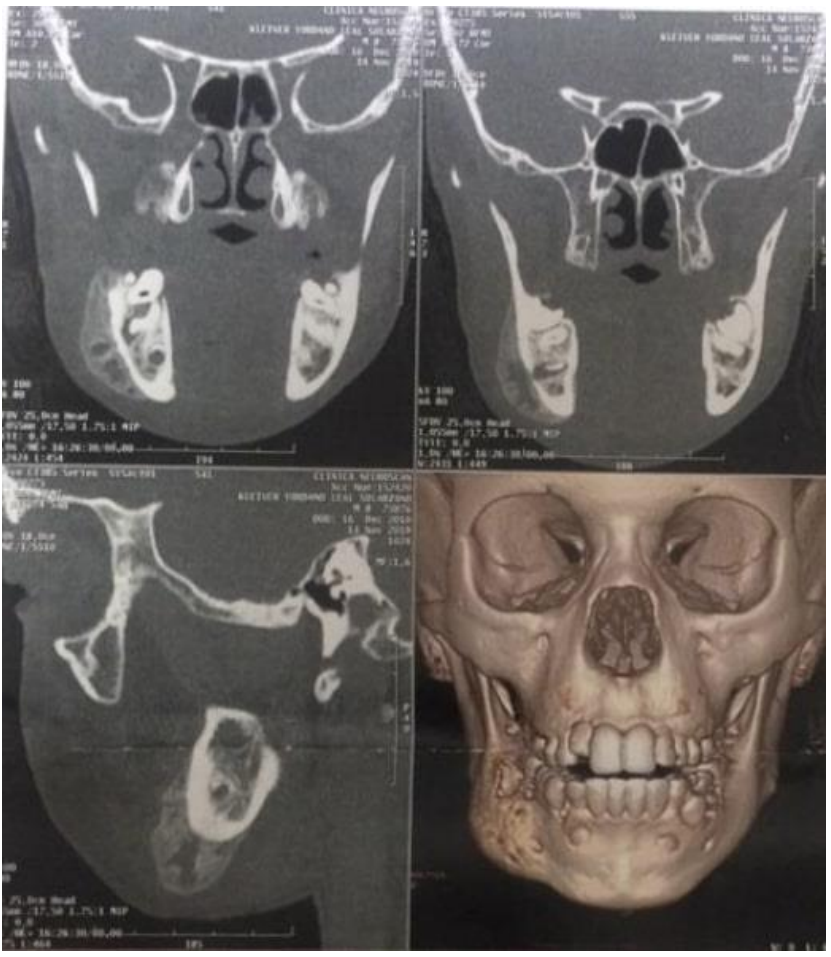

Figura 1: Tomografia computadorizada com reconstrução 3D (vista panorâmica e cortes sargital e axiais) demonstrando as lesões ósseas.

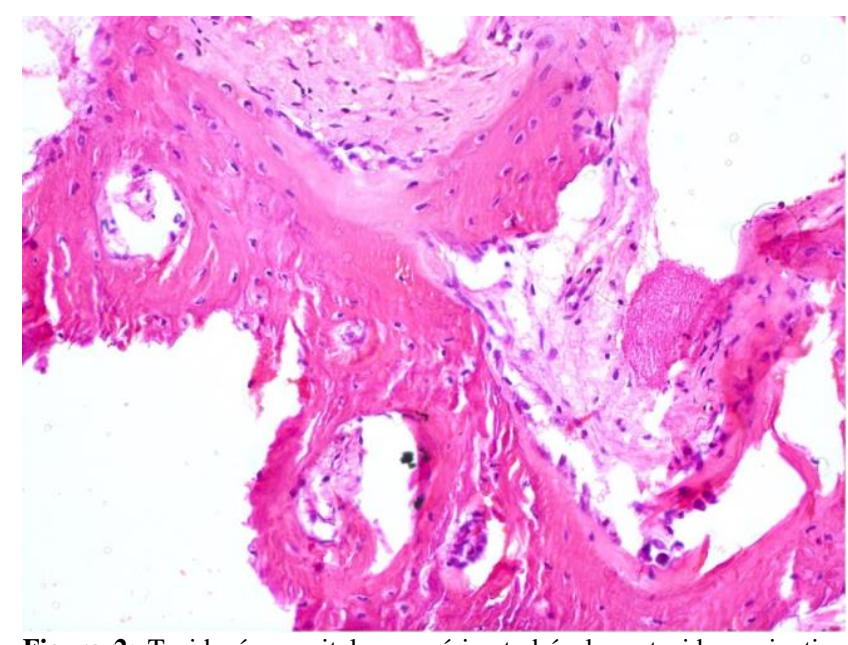

Figura 2: Tecido ósseo vital com várias trabéculas e tecido conjuntivo fibroso entremeando essas trabéculas. Intenso infiltrado inflamatório crônico.

Optou-se então pelo tratamento medicamentoso com Ceftriaxona, Hidrocortisona e Ibuprofeno associados à intervenção cirúrgica, que consistiu em desbridamento da lesão. No pósoperatório imediato o paciente respondeu bem, sem sintomatologia dolorosa intensa. Em acompanhamento, um ano após a intervenção cirúrgica não houve recidivas.

- Caso Clínico 2

Paciente do gênero feminino, 5 anos de idade, procurou o Hospital da Criança Santo Antônio 
com queixa de fortes dores em região mandibular. Ao exame clínico foi observado um processo inflamatório com sinais flogísticos, levando à hipótese diagnóstica de osteomielite, cisto aneurismático, linfoma de burkitt, osteosarcoma. Foi solicitada tomografia computadorizada (Figura 3), onde foi possível observar uma lesão abscedada de partes moles envolvendo a musculatura mastigatória (masseter) à esquerda, com área de flutuação profundamente junto à mandíbula estimada em 2,0 x $1,6 \mathrm{~cm}$ e com extensão superior envolvendo o músculo temporal até a base do crânio, onde há pequenas coleções. Adjacente a essas coleções havia uma lesão lítica no corpo da mandíbula à esquerda, junto à base do segundo pré-molar, apresentando contornos irregulares, sem zona de clivagem nítida com o osso normal, sem identificação também da cortical superior, medindo cerca de 1,0 x $0,6 \mathrm{~cm}$.

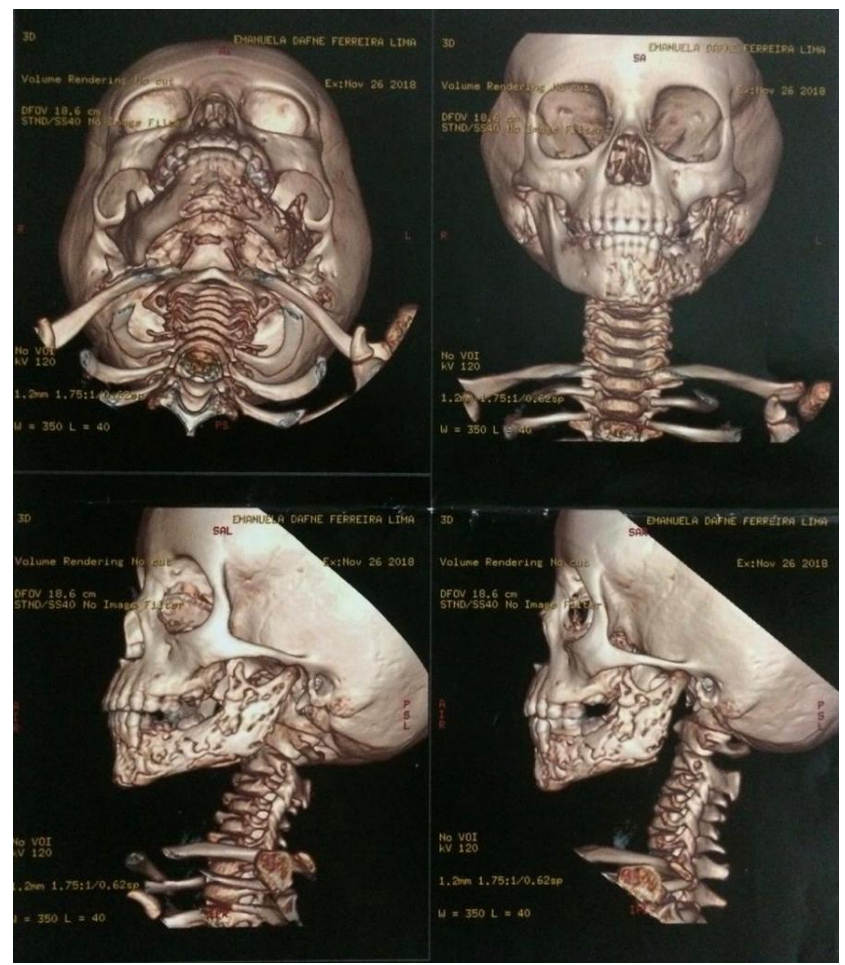

Figura 3: Reconstrução 3D (vista lateral, panorâmica e inferior) demonstrando lesões em tecido ósseo.

Foi realizada uma biópsia incisional com intenção diagnóstica. $\mathrm{O}$ exame anatomopatológico demonstrou fragmentos de tecido conjuntivo mostrando áreas de hialinização e discreta inflamação, como pode ser visto na Figura 4, além de fragmentos de tecido formado por trabéculas ósseas paralelas e com características de normalidade (Figura 5). Os aspectos clínicos e microscópicos favorecem o diagnóstico de osteomielite crônica. Foi instituído tratamento medicamentoso com Ceftriaxona, Hidrocortisona e Ibuprofeno associados à intervenção cirúrgica, que consistiu em desbridamento da lesão. No pós-operatório imediato o paciente respondeu bem, sem sintomatologia dolorosa intensa. Em acompanhamento, dois anos após a intervenção cirúrgica não houve recidivas.

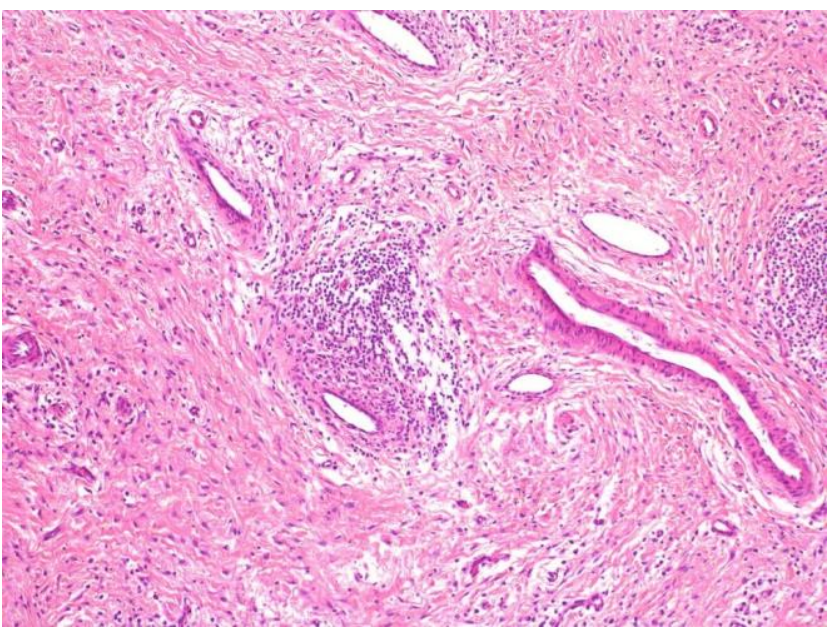

Figura 4: Fragmento de tecido conjuntivo com áreas de hialinização e discreta inflamação

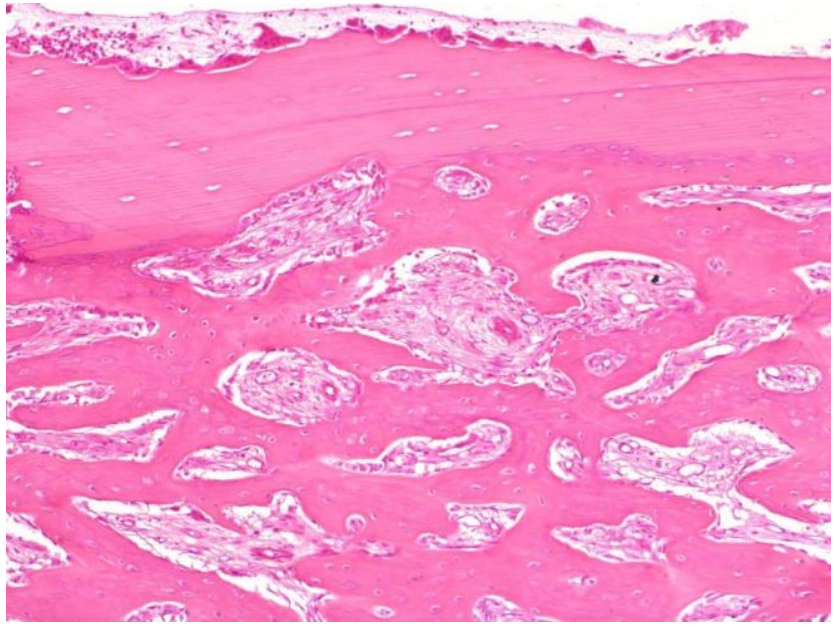

Figura 5: Fragmento de tecido formado por trabéculas ósseas paralelas, com características de normalidade.

\section{RESULTADOS E DISCUSSÃO}

Foi realizado levantamento de artigos científicos publicados nas bases de dados do Portal de Periódicos Capes, Google Acadêmico, Pubmed e Scielo. Na pesquisa, foram encontrados 39 artigos dos quais foram selecionados 18 para compor o trabalho. Nos casos relatados, os pacientes compareceram ao departamento de cirurgia e traumatologia bucomaxilofacial do HCSA apresentando queixas álgicas. Para fins diagnósticos foram solicitados exames de imagem $\mathrm{e}$ anatomopatológico. Diante dos dados obtidos, obteve-se o diagnóstico final, sendo esse osteomielite crônica. Para consubstanciar os resultados foi realizado levantamento de casos clínicos já existentes na literatura (Tabela 1), sendo possível comparar e analisar qual a melhor conduta em casos de osteomielite crônica em pacientes pediátricos, bem como estudar sua taxa de recidiva, evidenciando distintos fatores que caracterizam a patologia para fins diagnósticos e terapêuticos. Paim et. al. ${ }^{12}$ como Sousa et. al. ${ }^{13}$, demonstram que os exames tomográficos em três dos casos apresentaram evidências de perdas e lesões ósseas, no entanto, apresentam tipos distintos de osteomielite. Nos casos cinco e seis, os exames evidenciaram também lesões 
que envolviam a musculatura. Em todos os casos reportados os pacientes relataram queixas álgicas em sua anamnese, sendo evidenciado em quatro casos outros sinais característicos de inflamação.

Tabela 1. Análise de casos clínicos

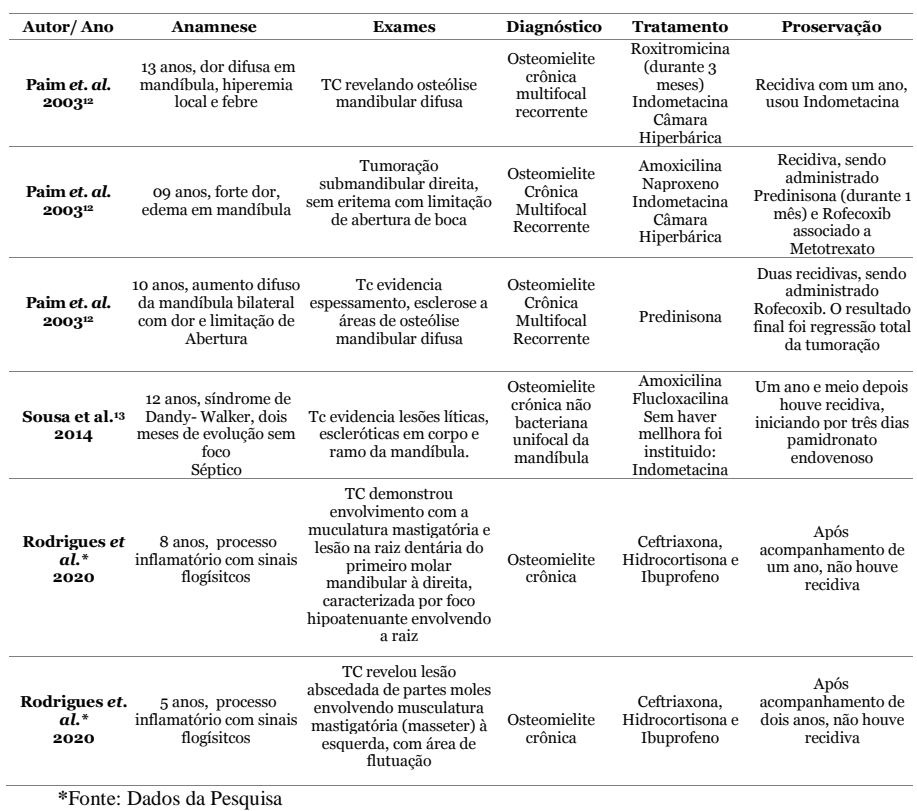

Para realizar o diagnóstico, todos os autores culminaram em realizar análises clínicas e de tomografias computadorizadas, no entanto, a importância e existência dos demais exames não devem ser desconsideradas. Com base no diagnóstico definido, foram apontados distintos tratamentos farmacológicos, sendo o uso de anti-inflamatórios não esteroidais prescrito em todos os casos. Em cinco dos seis casos houve a associação com antibióticoterapia, sendo o protocolo em pacientes com osteomielite. Em quatro dos seis casos houve relatos de recidivas do quadro, no entanto, três casos relatam resolução total do quadro. Em dois dos quatro casos que houve recidiva, o rofexoxib foi utilizado e eficaz no tratamento.

O diagnóstico e o tratamento da osteomielite crônica mandibular em pacientes infanto-juvenis representam um desafio devido às suas caracteristícas clínicas, podendo ser confundidos com outras patologias ${ }^{14}$. Entretanto, achados clínicos, radiográficos e histopatológicos demonstram características que permitem diagnosticar com precisão a osteomielite crônica, podendo ser tratada com êxito ${ }^{15}$.

A eficácia do tratamento inicial com antibióticos em pacientes portadores da osteomielite crônica mandibular é medida pela reposta clínica e alterações ósseas radiológicas ${ }^{14}$. Portanto, o retorno à aparência normal do osso em exames de imagens deve ser um sinal de resolução da condição ${ }^{9,14}$. Há casos como o relatado por Paimi et. al. ${ }^{12^{3}}$ em que a terapêtica inicial realizada com anti-inflamatório foi suficiente para o tratamento. No entanto, o sucesso da terapêutica através de distintos medicamentos deve ser atestado por meio do restabelecimento da estrutura óssea lesada.

Os antibióticos do tipo quinolonas se mostram como escolha por serem amplamente utilizados clinicamente devido à sua potente atividade antibacteriana, excelente penetração tecidual e alta biodisponibilidade $1^{6}$. No entanto, foi relatado que quinolonas induzem efeitos adversos no sistema musculoesquelético, incluindo cartilagem articular, ligamentos e tendões ${ }^{16,17}$.

Alguns autores afirmam que os antiinflamatórios não esteroidais (AINES) e os corticosteróides são úteis para manter o controle sintomático durante períodos de exacerbações 18 . Porém, nenhum estudo forneceu recomendações conclusivas sobre o uso dos AINES ou corticosteróides em pacientes portadores de osteomielite crônica mandibular ${ }^{18}$.

\section{CONSIDERAÇÕES FINAIS}

Pôde-se concluir que a associação de características clínicas, exames histopatológicos e achados radiográficos podem reunir características comuns para diversos tipos de osteomielite. A terapêutica adequada é estabelecida após o diagnóstico definitivo, sendo realizada principalmente por meio de terapia medicamentosa com antibióticos, além de anti-inflamatórios (esteroidais ou não-esteroidáis), bisfosfonatos ou imumoduladores. Em ambos os casos relatados, o tratamento foi eficaz utilizando terapia medicamentosa com o uso de anti-inflamatórios e antibióticos aliados ao tratamento cirúrgico, que consistiu em debridação da lesão. Ambos os pacientes foram acompanhados e prosservados, não havendo recidiva.

\section{REFERÊNCIAS}

1. Hupp JR, Ellis E, Tucker MR. Cirurgia oral e maxilofacial contemporânea. Rio de Janeiro: Elsevier; 2011.

2. Lew DP, Waldvogel FA. Osteomyelitis. Lancet. 2004;364(9431):369-79.

3. Baltieri BR, Gabrielli MAC, Gabrielli MFR, Pereira Filho VA, Lopes FS, Leite VA. Osteomielite em mandíbula de criança. Rev Odontol Unesp. 2014;43(N Especial):262.

4. Gaetti Júnior E, Gaetti Jardim EC, Faverani LP, Landucci KC, Landucci LF. Osteomielite crônica dos maxilares: aspectos clínicos, terapêuticos e microbiológicos. Salusvita. 2008;27(1):125-39.

5. Carek PJ, Dickerson LM, Sack JL . Diagnosis and management of osteomyelitis. Am Fam Physician. 2001;63(12):2413-20.

6. Miloro M, Ghali GE, Larse PE, Waite PD. Princípios de cirurgia bucomaxilofacial de Peterson. 2ed. São Paulo: Santos; 2008. 
7. Neville BW, Damm DD, Allen CM, Bouquot JE. Patologia oral e maxilofacial. Rio de Janeiro: Elsevier; 2011.

8. Watanabe $\mathrm{T}$, Ono $\mathrm{H}$, Morimoto Y, Otsuki Y, Shirai M, Endoh A et al. Skull involvement in a pediatric case of chronic recurrent multifocal osteomyelitis. Nagoya J Med Sci. 2015;77(3):493-500.

9. Suei Y, Taguchi A, Tanimoto K. Diagnosis and classification of mandibular osteomyelitis. Oral Surg Oral Med Oral Pathol Oral Radiol Endod. 2005;100(2):207-14.

10. Masocatto DC, Oliveira MM, Mendonça JCG. Osteomielite crônica mandibular: relato de caso. Arch Health Invest.2017;6(2):48-52

11. Ferraria N, Marques JG, Ramos F, Lopes G, Fonseca JG, Neves MC. Osteomielite crónica multifocal recorrente: série de 4 casos clínicos tratados com bifosfonatos. Acta Reumatol Port. 2014;39(1):38-45

12. Paim LB, Liphaus BL, Rocha AC, Castellanos LZA, Silva CAA. Osteomielite crônica multifocal recorrente da mandíbula: relato de três casos. J Pediatr. 2003;79(5):467-70.

13. Sousa MV, Malheiro R, Neves J, Varandas L, Conde M. Osteomielite crónica não bacteriana unifocal da mandíbula. Acta Reumatol Port. 2014:39;94-95

14. Kadom N, Egloff A, Obeid G, Bandarkar A, Vezina G. Juvenile mandibular chronic osteomyelitis: multimodality imaging findings. Oral Surg Oral Med Oral Pathol Oral Radiol Endod. 2011;111(3):e38-43.

15. Theologie-Lygidakis N, Schoinohoriti O, Iatrou I. Surgical management of primary chronic osteomyelitis of the jaws in children: a prospective analysis of five cases and review of the literature. Oral Maxillofac Surg. 2011;15(1):41-50.

16. Wang L, Wu Y, Tan Y, Fei X, Deng Y, Cao H et al. Cytotoxic effects of the quinolone levofloxacin on rabbit meniscus cells. J Appl Toxicol. 2014;34(8):870-77.

17. Deng Y, Chen B, Qi Y, Magdalou J, Wang H, Chen L. The effects of levofloxacin on rabbit anterior cruciate ligament cells in vitro. Toxicol Appl Pharmacol. 2011;257(1):67-73.

18. Obel G, Krogdahl A, Thygesen T, Godballe C. Juvenile mandibular chronic osteomyelitis: 3 cases and a literature review. J Oral Maxillofac Surg. 2013;71(2):305-9.

\section{CONFLITO DE INTERESSES}

Os autores declaram não haver conflitos de interesse.

\section{AUTOR PARA CORRESPONDÊNCIA}

\section{Matheus Francisco Barros Rodrigues}

matheusfbr08@outlook.com

Submetido em 14/05/2020

Aceito em 13/07/2020 